\title{
On Some Applications of Fuzzy Algorithm in Man- Machine Communication Research
}

\author{
D. DUTTA MAJUMDER (Membel) AND S. K. PAL*
}

(Mamuscript received on 20 February 1976)

\begin{abstract}
Present paper eununciates some practical applications of fuxzy set theory in problems of man-machine communication. Problems are recognttion of vowel speech sounds and identification of speakers from spoken words. Data used are derived from acoustic-phonetic and spectrographic analysis of large number of Hindi and Telugu (two of the major Indian Languages) speech sounds. Il is explained that Fuzzy set theory provides a suitable algorithm which is substantlvely different from the conventional quadtitative methods of system analysis yet presents an approximate but effective means of describing the behaviour of systems which are too ill-defined for precise mathematical analysis. In this paper, two decision algorithmic methods developed from fuzxy set theory and applied for machinc recognition of vowels and identification of speakers uith Telugu and Hindi speech sounds are presented along with the results of experiments.
\end{abstract}

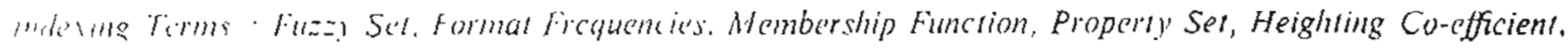
Simifanty l'etror, Panton Recognition, Recognition Score

$\mathrm{P}$ RESENT paper is a part of the work being conducted on min-machine compunuication iesearch (Dula Majumdar at al. 1968a, 1) visb, 1969, 1975, 1976, Dutta 1974a, $19746)$ at the Electronics and Communication Science 1)epul. of Indian Statistical Institute. There .t1 birrous approaches leading to the machine secognition of patterns as suggested by differcit authors (Duta Majumder ot al. 1975. 1)ula 1974a, Sebestyen 1962, Fu 1968). But most in the situations in the field of natural and social viences are too ili-defined to be analysed. To study wich complex behaved systems, the concept of fuzzy $x t$ and its subsequent developments in decision processes (Zadeh 1965, 1973) could be applied to a rcamonable extent. This algorithm is approximate but provides an eflective and more flexible basis for analysis of systems which are not precisely defined.

This paper would first of all, present briefly an introduction to fuzzy sets with associated operations, then describe two methods for classificatory analysis on the basis of fuzzy algorithm and finally their implementation on somc practica\} problems. First method is based on computation of weighted distance function and second method is based on computaturn of the similarity vectors of the property sets. Firstly, machine recognition of vowel speech sounds hosth for the Telugu and Hindi spoken words and scondly, speaker identification using the same data were experincuted with. But the recognition algoIulm for speaker identification could be further

* Depur of Electronics \& Comumunication Science, Indian Slitustical Institute, Calcutia 700035. extended to any number varying in sex and age. All the data processing was conducted on the ElecLronics Digital Computer Honeywell 400.

\section{FUIZY SET AND ITS OPERATIONS}

A class of events $x_{1}, x_{2}, \ldots, \ldots, x_{n}$ in the universe of discourse $U$ is defined as a fuzzy set $(A)$, if their transition from membership to non-membership is continuous rather than abrupt. $x_{1}, x_{2}, \ldots, x_{n}$ represent the supports of $A$ at which the value of nembership function $\mu_{A}(x)$ characterising the grade of membership of $x$ in $A$ is positive ranging between zero and one. A fuzzy set $A$ with the finite number of supports could therefore be viewed as

$$
A=\left\{\left(\mu_{A}\left(x_{i}\right), x_{i}\right)\right\} .
$$

in summation form,

$$
A=\mu_{1} / x_{1}+\mu_{2} / x_{2}+\ldots \ldots+\mu_{m} / x_{n}
$$$$
\text { or, } A=U_{i}\left(\mu_{i} / x_{i}\right), i=1,2, \ldots \ldots, n
$$

where + sign stands for the union of supporting points, rather than arithmetic sum. pes being the grade of membrship of $x_{1}$ in $A$ denotes the degree to which an event $x_{i}$ may be a member of $A$ or belong to $A$, and as it approaches unity, the grade of nembership of $x_{6}$ in $A$ becomes higher. For example, $\mu_{A}\left(x_{1}\right)=1$ indicates strictly the containment of the event $x_{i}$ in $A$ and if $x_{i}$ on the other hand does not belong to $A, \mu_{A}\left(x_{i}\right)=0$. Any intermediate value would represent the degree upto which $x_{i}$ could be a member of $A$.

If the support of a fuzzy set is only a single point $x_{t} \in U$, then

$$
A=\mu_{1} / x_{1}
$$


is called fuzzy singleton. Thus $A=1 / x_{1}$ for $\mu_{1}=1$ would obviously denote a non-fuzzy singleton. Basic operations related to fuzzy sets $A$ and $B$ having membership values $\mu_{A}(x)$ and $\mu_{B}(x)$ respectively are summarized here.

(i) $A$ is equal to $B(A=B)$

$\Rightarrow \mu_{A}(x)=\mu_{B}(x)$, for $\forall x$

(ii) $A$ is complement of $B(A=7 B)$

$\Rightarrow\left(i-\mu_{B}(x)\right)=\mu_{A}(x)$, for $\forall x$

(iii) $A$ is subset of $B(A \subset B)$

$\Rightarrow \mu_{A}(x) \leqslant \mu_{B}(x)$, for $\forall x$

(iv) Union of $A$ and $B(A \cup B)$

$\Rightarrow \mu_{A U_{B}}(x)=V\left[\mu_{A}(x), \mu_{B}(x)\right]$, for $\forall x$ $\checkmark$ denotes naximum

(1) Intersection of $A$ and $B(A \cap B)$

$\Rightarrow \mu_{A} \cap_{B}(x)=\wedge\left[\mu_{A}(x), \mu_{B}(x)\right]$. for $\forall x$. $\wedge$ denotes minimum

(ii) Product of $A$ and $B(A B)$

$\Rightarrow \mu_{A B}(x)=\mu_{A}(x) \mu_{B}(x)$, for $\forall x$.

In addition to the above mentioned operations, there are other operations applied on fuzzy set $A$. These are,

(i) Concentration of $A(\operatorname{CON}(A))$

$\Rightarrow \mu \operatorname{con}(A)(x)=[\mu A(x)]^{2}$, for $\forall . r$

(ii) Dilation of $A$ (DIL (A))

$\Rightarrow \mu_{D / L(A)}(x)=\left[\mu_{A}(x)\right]^{1 / 2}$, for $\forall x$

iii) Contrast Intensification of $A(I N T(A))$

$\Rightarrow \mu_{I N T(A)}(x)=\left\{\begin{array}{l}2\left[\mu_{A}(x)\right]^{2}, 0 \leqslant \mu_{A}(x) \leqslant 0.5 \\ {\left[1-2\left(1-\mu_{A}(x)\right)^{2}\right], 0.5 \leqslant \mu_{A}(x) \leqslant 1.0 .}\end{array}\right.$

All these operations have the effect of altering the fuzziness of a set. Effect of DIL $(A)$ is opposite to that of concentration which reduces the magnitude of $\mu_{A}(x)$ in relatively smaller amount for those $x$ having higher membership value in $A$ compared to those with low $\mu_{A}$ value. Contrast intensification as its name implies, reduces the fuzziness of $A$ by increasing the value of $\mu_{A}(x)$ which are above 0.5 and decreasing those which are below it.

\section{DECISION ALGORITHM}

Consider an $N$-dimensional feature vector space $\Omega_{x}$ containing $m$ ill-defined pattern classes $C_{1}, C_{2}$, $\ldots C_{b}, \ldots C_{n}$ to be recognized with defined set of $N$-dimensional prototypes $R_{1}, R_{2}, \ldots R_{1}, \ldots R_{m}$ such that

$$
R_{j}^{(i)} \in R_{d}
$$

$l=1,2, \ldots h_{j} ; h_{f}$ is the number of reference vectors in set $R_{f}$.

Present classification model aims to assign an unknown event with $N$ number of features

$$
X==\left[\begin{array}{c}
x_{1} \\
x_{2} \\
\vdots \\
x_{n} \\
\vdots \\
x_{N}
\end{array}\right]
$$

in $\Omega_{x}$, to its appropriate class $C_{3}$ for which its $\mu$-value $\mu_{1}(x)$ or magnitude of the similarity vector $S_{f}(x)$ possesses maximum value where $x_{n}$ represents the $n$th measured feature the event has.

\section{Mcthod I}

If $W_{\text {in }}$ (having magnitude less than or equal to unity) and $R_{\text {,n }}$ associated with $j$ th class represent the magnitude of weighting co-efficient and reference feature respectively for $n$th component and $x_{i, n}$ is the numerical value of $n$th component for $i$ th token in $C_{1}$ containing $M$ no. of events such that

$$
\begin{aligned}
& W_{i n}{ }^{2}=\frac{1}{\frac{1}{M} \sum_{i=1}^{M} x_{i, n}{ }^{2}-R_{n^{2}}{ }^{2}} \\
& R_{i n}=\frac{1}{M} \sum_{i=1}^{M} x_{i, n} .
\end{aligned}
$$

Then membership function of an unknown pattern $X$ corresponding to $j$ th class could be expressed as

$$
\mu_{f}(x)=\left[1+\left[\begin{array}{c}
\left.d(x, R,)^{\prime}\right) \\
E
\end{array}\right]^{F}\right]^{-1}
$$

where $E$ is an arbitrary positive constant and $F$ is any integer. These constants are included only to affect the fuzziness of a set (Dutta Majumder and Pal 1976). Here $d\left(X, R_{1}\right)$, the distance between $X$ and prototype $R_{1}$ is defined $\dot{T}$ as the simallest of the distances between $X$ and each reference vector $R_{i}^{(l)}$ in $R_{i}$. Thus

$$
\begin{aligned}
d\left(X, R_{j}\right) & =\Lambda_{1}\left|X-R_{t}^{(+)}\right| \\
\text {and }\left|X-R_{1}{ }^{\prime \prime}\right| & =\left[\sum_{n=1}^{N}\left[W_{m n}\left(X_{n}-R_{m n}{ }^{(I)}\right)\right]^{2}\right]^{1 / 2}
\end{aligned}
$$

where $\mid-1,2, \ldots \ldots \ldots \ldots \ldots, h_{1}$

$$
j=1,2, \ldots \ldots \ldots \ldots \ldots, m \text {. }
$$

Equation (15) defines the grade of membership of $X$ in $C_{j}$ and its $\mu$-value would be 1 for $d\left(X, R_{j}\right)=$

0 . Thus an unknown pattern $X$ could be identified to be a nember of $k$ th class if $\mu_{k}(X)$ is maximum i.e.

if $\mu_{k}(x)=V\left[\mu_{j}(x)\right]$, decide $X \in C_{k}$

$j$ and $k$ may have any integer value from 1 to $m$.

\section{Method II}

Let $p_{p}, p_{2}, \ldots \ldots \ldots, p_{n} \ldots \ldots \ldots \ldots, p_{N}$ be the $N$ properties each of which represents some aspects of the unknown pattern $X$ and has value only in the interval $[0,1]$ such that

$$
P_{n}=\left[1+\frac{\left|\bar{x}_{n}-x_{n}\right|^{F}}{E}\right]^{-1}
$$

TThis is one of the most accepted definitions of the distance function used in statistical classificatory analysis (Rro 195?, Sebestyen 1962). 
where $\bar{x}_{n}=v_{i} \frac{1}{h} \sum_{l=1}^{h} x_{n}{ }^{n}$

It no. of reference vectors in a class. Constants $E$ and $F$ have the same effects as in the previous method in affecting the fuzziness of a set.

There are $h$ no. of reference prototypes in a class $C$ and each prototype may be represented as

$$
\begin{aligned}
& R_{1}^{(l)}=\left\{\begin{array}{cccc}
(l) & (l) & \ldots, p_{n t}{ }^{(l)} \ldots \ldots p_{N 1}{ }^{(l)}
\end{array}\right\} \\
& l=1,2, \ldots \ldots h \\
& j=1,2, \ldots \ldots \ldots
\end{aligned}
$$

$P_{n f}(1)$ denotes the property $p_{n}$ of the 7 th prototype in $C_{3}$. Then the similarity vector $S_{f}(X)$ for the pattern $X$ with respect to $j$ th class is

$$
s_{1}(x)-\left\{s_{1}, s_{2 y}, \ldots \ldots, s_{n d} \ldots \ldots, s_{N}\right\}
$$

"here numerical value of $s_{n}$, denoting the degree uplo which $m$ th property of $\lambda$ resembles with that of Ah chas is given by

$$
\text { wherc } s_{r j}=1 \sum_{h=1}^{h} s_{n j}(h
$$

where

$$
s_{n i}^{(1)}=\left[1+A\left|\begin{array}{c}
p_{n}(b) \\
p_{n 3}^{(b)} p_{n}
\end{array}\right|\right]^{--1} .
$$

4 is any positive constant. Thus, the grade of membership of an unknown pattern $\lambda-\left\{p_{1}, p_{2}\right.$

$\left.\ldots p_{n} \ldots \ldots p_{N}\right\}$ with reference to all the classes are found from the magnitudes of their correspondhag similarity vectors and decide it to belong to $C_{k}$ if.

$$
\begin{array}{ll}
\because & S_{n g},-S_{n k} \\
\because & 1,2, \ldots \ldots \ldots, N \\
k .1 & 1,2 \ldots \ldots \ldots \ldots, m .
\end{array}
$$

\section{MPLEMENTATION OF THE THEORY AND EXPERIMENTAL RESULTS}

To demonstrate the application of the theory to the problem of vowel speech sound and speaker recognition, vowel formant frequencies $F_{1}, F_{2}$, and $F_{3}$ were extracted from selected Telugu and Hindi speech samples in CNC combination (Dutta Majulnder et al. 1974, 1973). There are 10 sets of Telugu vowels which were transformed to 6 classes which are phonetically non-identical namely. $/ \partial /$, $\{a \%, I J,|U|,|E|$ and $|O|$ such that

$$
\begin{aligned}
& C_{1} \supset C_{1}^{1-}, C_{4}: \\
& C_{U} \supset C_{u^{-1}}, C_{u}: \\
& C_{E} \supset C_{0}^{1-}, C_{0}: \\
& C_{0} \supset C_{0}{ }^{-1}, C_{0}:
\end{aligned}
$$

where shorter and longer subsets being phonetically alike are treated in the same class.

Thus in the present problem, $N=3, j$ has maximum value of 6 and $h$ equals 1 for $C_{0}$ and $C_{n}$ : and 2 for $C_{1}, C_{U}, C_{E}$ and $C_{0}$.

Table 1 shows typical values of formants for each vowel. Let us consider for example, the sample of class [I] whose $d\left(X, R_{f}\right)$ values are

$$
\begin{aligned}
& \text { For Class }[\hat{o}]=4.93 \\
& {[a:]=11.67} \\
& {[l]=\wedge[1.08,1.80]=1.08} \\
& {\left[U^{\prime}\right]=\wedge[8.64,13.57]=8.64} \\
& {[E]=\wedge[2.67,1.57]=1.57} \\
& \text { and } \quad[O]=\Lambda[8.11,18.80]=8.11
\end{aligned}
$$

Corresponding $\mu$-values with $E=F=1$ are found from eqn. (15) to be $0.17,0.08,0.48,0.10,0.39$ and $0.1 \mathrm{f}$ respectively and verify its containment in $C_{r}$. Besides, these values defining the degree to which it

\begin{tabular}{|c|c|c|c|}
\hline Vowcl class & $F_{1}\left(\mathrm{H}_{\gamma}\right)$ & $F_{ \pm}(\mathrm{Hz})$ & $F_{2}(\mathrm{~Hz})$ \\
\hline$|a|$ & 550 & 1550 & 2400 \\
\hline 10:1 & 700 & 1200 & 2550 \\
\hline $\mid \mathrm{II}$ & 400 & 2250 & 2600 \\
\hline$|U|$ & 350 & 0850 & 2600 \\
\hline$|E|$ & 500 & 1900 & 2650 \\
\hline $10 i$ & 500 & 0950 & 2600 \\
\hline
\end{tabular}
resembies the membership of the class to which it belongs other possible order of classes is indicated to be $/ E /, / \mathrm{a} /, / U /, / \mathrm{O} /$ and $/ a ; /$. This serialized result

\begin{tabular}{|c|c|c|c|c|c|c|}
\hline $\begin{array}{l}\text { Classified } \\
\text { as }\end{array}$ & & & 侟 & & & \\
\hline $\begin{array}{l}\text { as } \\
j a /\end{array}$ & $\begin{array}{r}10 \\
52\end{array}$ & $\begin{array}{r}7 . / 7 \\
17\end{array}$ & $U_{i}$ & i & is & $\begin{array}{r}18 \\
7\end{array}$ \\
\hline la: & 8 & 70 & & & & \\
\hline$[1 /$ & & & 155 & & 25 & \\
\hline $\mid \mathrm{U} /$ & 1 & & & 144 & & 34 \\
\hline$|E|$ & 9 & & 17 & & 154 & 1 \\
\hline $10 !$ & 2 & 2 & & $G$ & 13 & 138 \\
\hline $\begin{array}{l}\text { Recognition } \\
\text { Score }(\%)\end{array}$ & 72.2 & 78.65 & 90.1 & 95.36 & 74.4 & 76.66 \\
\hline
\end{tabular}
is similar to their phonetical order.

Again membership values computed on formants of vowel /a/ (Table 1) come to be $\mu_{\mathrm{a}}=0.24, \mu_{\mathrm{a}}$ $-0.17, \mu_{I}=0.13, \mu_{U}=0.28, \mu_{E}=0.23$ and $\mu_{0}=0.54$ and decide the classes in order of possibility as $/ O /,|U /|, E /,|O /| a:, /$ and $|J|$ which is almost correct from the same point of view.

There are about 900 such samples whose proper classes were assigned exactly in same way and results are tabulated in Table 2. Maximum number of misrecognized samples for vowels $|l /| E,|,| \partial /, \mid a ; /$, $|U|$ and $\mid O /$ as expected correspond to classes $|E|$, $|I /| a:, \%, 10 /, 1 O /$ and $/ O /$ respectively.

\section{TABLE $1 \ldots$ TYPICAL VALUES OF FORMANT FREQUENCIES}

TABLE 2--RECOGNITION SCORE FOR TELUGU VOWELS Actual Vowel Class 
TABLE 3--RECOGNITION SCORE FOR HINDI VOWELS Actual Vowel Class

Classified

as

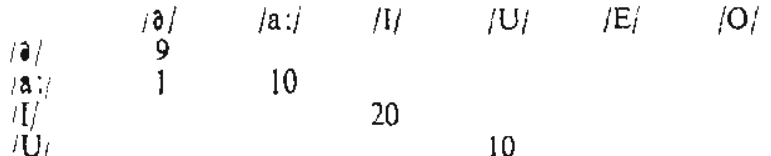

$\mathrm{E}$

101

Recognition

Score $(\%) 90.0$

10

20
TABLE 4 - TYPICAL VALUES OF FORMANT FREQUENCIES FOR HINDI VOWEL [i:] UTTERED BY THREE SPEAKERS AND THEIR RECOGNITION RATE

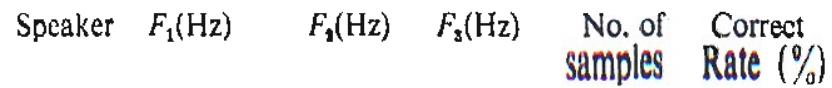

\begin{tabular}{rrrrrr}
$X$ & 350 & 2350 & 2800 & 38 & 89.47 \\
$Y$ & 350 & 2250 & 2700 & 66 & 90.90 \\
$Z$ & 300 & 2350 & 3000 & 66 & 100.00 \\
\hline
\end{tabular}

Hindi vowels consist of 8 classes $\left(1 \partial /,|a \%,| i^{1}-|| i \%\right.$, $\left|u^{-1} /,\right| u,|| E \mid$, and $\left.\mid O /\right)$ and number of samples were only 10 for each class. As in the Telugu vowels longer and shorter subsets were pooled together in the same category and recognition rate obtained by this method is shown in Table 3.

Second recognition scheme described in the last section was applied to the problem of speaker identification. Typical formant frequencies for vowel [i] uttered by 3 informants $X, Y$ and $Z$ say, in the age group of $28 / 30$ years are tabulated in Table 4 .

As the third formant $F_{3}$ is more significant compared to others for bearing information about sex and age of an informant, the recognition parameters choosen are $\left(F_{3} / F_{1}\right),\left(F_{3} / F_{2}\right)$ and $F_{3}$. In this problem, $N=3, m=3$ and for reference prototypes in each class $h=5$. For example, consider the feature of informant $Y$ whose associated properties (eqn. 18) are

$$
\begin{aligned}
& p_{1}=0.9994626 \\
& p_{2}=0.9999990 \\
& p_{3}=0.0588235
\end{aligned}
$$

where $\bar{x}_{1}=10.033, \bar{x}_{2}=1.3, \bar{x}_{8}=3100, E=100$ and $F=2$.

Using equations (22) and (23) and taking constant $A$ to be inverse of the standard deviation of the prototype features, the magnitudes of the similarity vectors for this pattern with reference to three classes are found to be $2.50,2.986$ and 2.965 respectively which indicate its appropriate containment. Present procedure was repeated entirely in the same fashion for the other events and net rate of identifications are mentioned in Table 4.

\section{CONCLUSION}

Use of fuzzy set to the classification problem of imprecisely defined patterns is experimented for computer recognition of speakers and spoken vowels. Knowledge of weighting co-efficients and reference vectors used in this paper could be available also from any size of samples selected within a class. For Telugu speech sounds, since vowel recognition is done irrespective of speakers, its net recognition rate is lowered as compared to Hindi vowels.

In the problem of speaker identification, the machine was trained for each informant by 5 samples which can be changed to any size to have improved score. Further work on this algorithm and classification of a number of male and female speakers in the different age groups is in progress.

\section{ACKNOWLEDGEMENT}

Authors wish to express their thanks to Prof, C. R. Rao, FRS, Director of Indian Statistical Institute for his kind interest in the work and also to the Council of Scientific and Industrial Research for Awarding fellowship to Mr. S. K. Pal, one of the authors.

\section{REFERENCES}

1. Dutta Majumder (D) and DutTa (AK). a. Some Studics on Automatic Speech Coding and Recognition Procedure. Ind. Jour. Phys. 42, 1968; 425-443.

2. Dutta Majumder (D) and DutTa (AK). b. A Model for Spoker word Recognition. Proc. Automazione Estrumentazione. Milan, Italy, 1968; 249-259.

3. Dutta Maudmder (D) and DuTta (AK). An Analyser Coder for Machine Recognition of Human Speech. JITE. 15,$4 ; 1969 ; 233-243$.

4. Dutra Majumber (D), Dutra (AK) and Pal (SK). Computer Recognition of Vowel Speech Sounds Using Three-dimensional Weighted Discriminant Function. J. CS1. 7, 1; 1976.

5. Dutra Majumder (D) and PaL (SK). The Concepts of Fuzzy Sets and its application in Pattern Recognition Problems. Proc. Eleventh Annual Symp. of the Computer Soc. of India. Hyderabad, 1976.

6. DutTA (AK). a. An Experimental Procedure for Handwritten Character Recognition. IEEE Trans. C-23, 5; $1974 ; 536-545$.

7. DutTa (AK). b. Computer Recognition of Handwritten Roman Cursive Script. Proc. IEEE International Symp. on Information Theory. Notre Dame, Indiana, USA, 1974.

8. Sebestyen (GS) Decision Making Procedures in Pattern Recognition. 1962. The Macmillan Co., NY. P 8-82.

9. Fu (KS). Sequential Methods in Pattern Recognition and Machine Learning. 1968. Academic Press, London. P3-18.

10. ZADEH (LA). Fuzzy Sets. Inform. Control. 8; 1965; 338-353.

11. ZADEH (LA). Outline of a New Approach to the Analysis of Complex Systems and Decision Processes. IEEE Trans. SMC-3, 1; 1973; 28-44.

12. Dutta Majumder (D), Dutta (AK), Ganguly (NR), MUKHERJEe (B) and Sarkar (R). Studies on AcousticPhonetic Features of Telugu Speech Sounds. ECSL Series on Phonological Studies. Mahalanobis Memorial Issue, Nov. 1974.

13. DUtTa MajumDer (D), DUtTa (AK) and Ganguly (NR). Some Studies on Acoustic Features of Human Speech in relation to Hindi Speech Sounds. Ind. Jour. Phys, 47, 1973; 598-613.

14. RAo (CR). Advanced Statistical Method's in Rescarch. 1952. J. Wiley \& Sons. NY. 